Cite this: J. Mater. Chem. A, 2013, 1, 12971

Received 14th March 2013 Accepted 11th September 2013

DOI: 10.1039/c3ta80001f

www.rsc.org/MaterialsA

\section{Using POSS reagents to reduce hydrophobic character in polypropylene nanocomposites $\uparrow$}

\author{
Emily R. Smith, Brendan J. Howlin and Ian Hamerton* \\ Three POSS reagents (1,2-propanediolisobutyl POSS, glycidylisooctyl POSS, and triglycidylisobutyl POSS, all \\ $5 \mathrm{wt} \%$ ) are incorporated into a commercial isotactic polypropylene, PP. Infrared and Raman spectroscopy \\ show that the blending has been successful as evidenced by the presence of Si-O-Si bands at 1098- \\ $1110 \mathrm{~cm}^{-1}$, which are not present in the base polymer. The inclusion of $5 \mathrm{wt} \%$ of the various POSS \\ reagents leads to a general increase in the loss modulus at the $T_{\mathrm{g}}$ (e.g. the greatest increase being from \\ ca. $125 \mathrm{MPa}$ to ca. $150 \mathrm{MPa}$ ) from DMTA data, although the $T_{\mathrm{g}}$ of the PP is slightly diminished by $4-8 \mathrm{~K}$ \\ depending on the POSS used. The melting behaviour is also altered as the addition of POSS leads to a \\ more diffuse and multimodal melting endotherm in the blends, although the melting temperature does \\ increase slightly $(7 \mathrm{~K})$. TGA data confirm that the thermal and thermo-oxidative stability of PP is not \\ adversely affected by the inclusion of the POSS reagents at this concentration. Tensile data show that \\ the ultimate tensile strength (ca. $24.7 \pm 0.1 \mathrm{MPa}$ ) remains the same, although the tensile modulus \\ (ca. $1.24 \pm 0.06 \mathrm{GPa}$ ) is reduced by up to $0.22 \mathrm{GPa}$ and the maximum load does reduce by ca. 80-160 $\mathrm{N}$. \\ Contact angle measurements for the PP $\left(99.72^{\circ} \pm 0.73\right)$ show that the inclusion of the POSS reagents at \\ this level does reduce its hydrophobic character as the greatest depression in contact angle is effected \\ by the inclusion of $5 \mathrm{wt} \%$ triglycidylisobutyl-POSS (85.77 \pm 1.39 ). Molecular modelling and QSPR \\ techniques are used to predict selected physical properties of the PP/POSS nanocomposites.
}

\section{Introduction}

Polypropylene (PP) is one of the truly big players in the commodity polymer marketplace. In 2007, the global market for PP accounted for around 45.1 million tons and a turnover of $c a$. $\$ 65$ billion (47.4 billion $€$ ). ${ }^{1}$ The polymer is widely used in a variety of commodity and higher performance applications in bulk, film, and fibre forms, with a balance of desirable properties such as excellent resistance to attack from dilute and concentrated acids, alcohols, bases and mineral oils; good resistance to attack from aldehydes, esters, aliphatic hydrocarbons, ketones and vegetable oils. With a wide range of use temperatures $\left(0-135^{\circ} \mathrm{C}\right)$, a density of $0.9 \mathrm{~g} \mathrm{~cm}^{-3}$ and a melting temperature of $170{ }^{\circ} \mathrm{C}$ (melt flow index 0.9-1000 g/10 min depending on grade), the translucent rigid polymer is suitable for autoclave moulding. Typical tensile strength for the isotactic polymer is $4500 \mathrm{psi}$, making it suitable for housings for domestic appliances, extrusion of fibres and filaments for clothing and medical fabric and in generic bottle lids. With an alkylene backbone and methyl substituents the bulk polymer is hydrophobic with typical contact angles of $87.5^{\circ}-117^{\circ}$ being recorded for PP. ${ }^{2}$ As PP possesses an inherently low surface

Department of Chemistry, Faculty of Engineering and Physical Sciences, University of Surrey, Guildford, Surrey, GU2 7XH, UK.E-mail: i.hamerton@surrey.ac.uk

$\dagger$ This paper is dedicated to the memory of Mr Dennis Smith, MBE. energy, it can be rendered unsuitable in certain applications wherein for instance, the polymer needs to be coated with conventional polar paints or coatings (particularly more modern, low VOC water based systems). Consequently, the surface free energy would need to be increased in order for the material to adhere successfully and investigations have been made into making the PP surface more polar. One of the most common ways of modifying the surface free energy of PP is to use plasma treatment. While this is effective and can lead to reductions in contact angles from $c a .110^{\circ}$ to $c a .70^{\circ}$ after exposure to a particular plasma treatment for 150 seconds, ${ }^{3}$ it can leave surfaces with considerable surface roughness. If this is ultimately undesirable, the use of POSS reagents (polyhedral oligomeric silsesquioxanes) has been used to resolve this problem by using their physical bulk to 'fill' the surface imperfections and therefore generate a comparatively smooth surface. $^{4}$

As POSS reagents are typically structurally very different to the polymer within which they are incorporated, it is important to consider dispersion upon mixing. This is particularly prudent if the incorporation is purely physical entrapment, as opposed to reactive blending. It has been proposed that the POSS molecules migrate to the surface of the PP, as a result of the relative energies involved. The cohesive energy of the POSS molecule and the matrix chains are, in relative terms, lower than the cohesion energy of the polymeric chains, causing the 
POSS molecules to be effectively propelled to the surface of polymer. ${ }^{5}$ While there are few investigations into increasing the surface energy of PP by the utilisation of functionalised POSS, there are various examples of adopting this approach to other polymeric materials. For instance, trisilanolphenyl POSS has been shown to effect a small $\left(1 \mathrm{mN} \mathrm{m}^{-1}\right)$ increase in the surface energy of polystyrene at low concentrations (5-10 wt\%). ${ }^{6} \mathrm{Simi}-$ larly, the hydrophilicity of selected polyamides have increased by introducing up to $10 \mathrm{wt} \%$ POSS, with the finding that the contact angle was reduced from $(62.9 \pm 0.9)^{\circ}$ to $(38.6 \pm 0.4)^{\circ}$ with the highest loading. ${ }^{7}$ The aim of the present work is to examine the effects of introducing selected POSS reagents into the bulk polymer in order to reduce the hydrophobicity of the resulting nanocomposite, preferably without diminishing other physical and mechanical properties. Moreover, we attempt to explore whether a combination of QSPR (quantitative structure property relationships) and molecular simulation will enable us to predict the properties of these and related nanocomposites.

\section{Experimental}

\section{Materials}

The commercial metallocene grade isotactic polypropylene (PP) of density $0.900 \mathrm{~g} \mathrm{~cm}^{-3}$ and melt flow index $24.0 \mathrm{~g} / 10$ minutes (at load $2.16 \mathrm{~kg}, 230^{\circ} \mathrm{C}$ ) was used as received. The POSS reagents (1,2-propanediolisobutyl POSS AL0130; glycidylisooctyl POSS, EP0419; and triglycidylisobutyl POSS, EP0423, Fig. 1) were all supplied by Hybrid Plastics Inc., Hattiesburg, who undertook the initial blending in house. Both AL0130 and EP0423 are structurally related, containing a cubic inorganic cage structure with silicon atoms at the apices and oxygen atoms on the vertices. Both POSS reagents are monofunctional containing a glycidyl ether moiety (EP0419) or a 1,2-propanediol moiety (AL0130) bonded to one of the silicon atoms; the remaining apices bear either isooctyl (EP0419) or isobutyl (AL0130) moieties. In contrast, EP0423 represents a more open structure (from which the final closing silicon atom is missing to complete the cage). This triglycidyl ether contains isobutyl groups on the remaining silicon atoms. Blends of PP comprising $5 \mathrm{wt} \%$ of each POSS reagent were produced as described below.

Compounding of the PP/POSS blends. All compounding was carried out using a $25 \mathrm{~mm}$ counter rotating twin screw extruder (BP Processing), L/D $40: 1$. The compounding temperature zones ranged from 150 to $180{ }^{\circ} \mathrm{C}$. All extruded strands were passed through a water trough and pelletised/dried during palletisation. The AL0130 (Lot \# 042710, $0.15 \mathrm{~kg}$ ) and PP (2.85 $\mathrm{kg}$ ) were added to the extruder throat via weight loss feeders and $2 \mathrm{~kg}$ of PP-POSS material was made. Since EP0419 (Lot \# $09071073,0.15 \mathrm{~kg}$ ) is a liquid it was tumble mixed with PP pellets to achieve uniform coating of pellets. The resulting mixed material was then added to the extruder using a weight loss feeder and compounded, dried, pelletized as above. Since EP0423 (Lot \# 06291029, $0.15 \mathrm{~kg}$ ) is a liquid it was tumble mixed with PP pellets $(2.85 \mathrm{~kg})$ to achieve uniform coating of pellets. The resulting mixed material was then added to the extruder using a weight loss feeder and compounded, dried, pelletized as above.

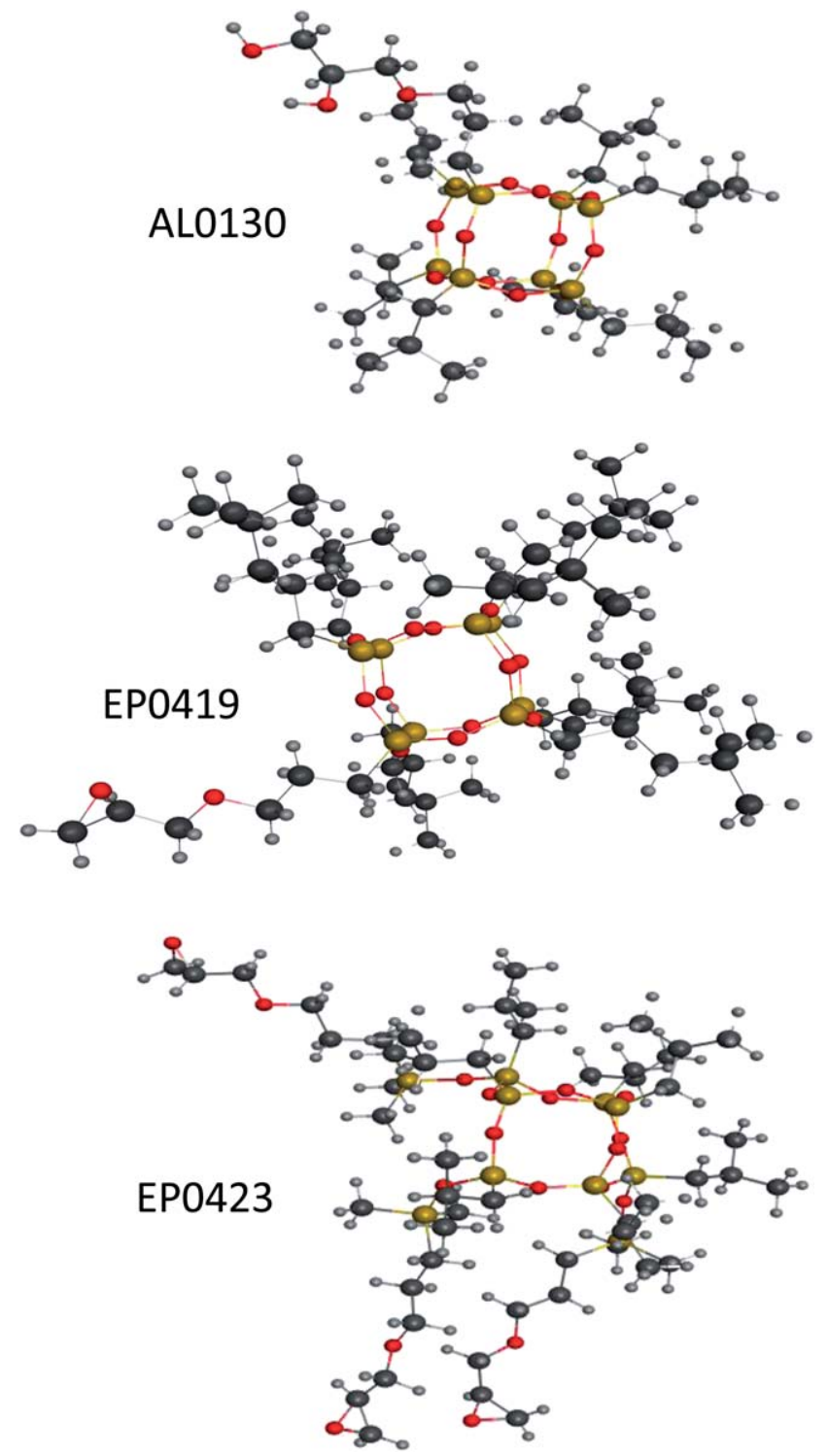

Fig. 1 Representations of POSS reagents used in this work (structures produced using MOE 2010.11). N.B., carbon atoms are dark grey, hydrogen atoms are light grey, oxygen atoms are red and silicon atoms are golden.

\section{Apparatus}

Fourier transform infrared spectra were recorded using a Perkin-Elmer system 2000 spectrometer. The samples (thin films) were presented in the ATR module. In each case 16 scans, at a resolution of $4 \mathrm{~cm}^{-1}$, were recorded (4000$550 \mathrm{~cm}^{-1}$ ) and co-added to produce the final spectrum. Raman spectra at a resolution of $1.0 \mathrm{~cm}^{-1}$ were acquired on the same spectrometer at room temperature using a Nd-YAG laser (excitation wavelength $1064 \mathrm{~nm}, 810 \mathrm{~mW}$ ).

Sample alignment was monitored using the sulphur band at $217 \mathrm{~cm}^{-1}$. Samples were presented in Durham tubes, having filled these with molten PP.

Differential scanning calorimetry (DSC) was undertaken using a TA Instruments Q1000, on samples (cut thin film samples) of $5 \pm 0.5 \mathrm{mg}$ in hermetically-sealed aluminium pans. 
Experiments were conducted at a heating rate of $10 \mathrm{~K} \mathrm{~min}^{-1}$ from room temperature to $300{ }^{\circ} \mathrm{C}$ under flowing nitrogen $\left(50 \mathrm{~cm}^{3} \mathrm{~min}^{-1}\right)$. The samples were then cooled at $10 \mathrm{~K} \mathrm{~min}^{-1}$ from 300 to $20{ }^{\circ} \mathrm{C}$ before a rescan was run at $10 \mathrm{~K} \mathrm{~min}^{-1}(25-$ $\left.300{ }^{\circ} \mathrm{C}\right)$ to reveal glass transition temperature $\left(T_{\mathrm{g}}\right)$ for samples (taken as the midpoint of the transition).

Thermogravimetric analysis (TGA) was undertaken using a TA Instruments Q500, on samples of $9 \mathrm{mg}$ in a platinum crucible. Experiments were conducted at a heating rate of $10 \mathrm{~K}$ $\min ^{-1}$ from room temperature to $700{ }^{\circ} \mathrm{C}$ under nitrogen (balance gas, nitrogen: $40 \mathrm{~cm}^{3} \mathrm{~min}^{-1}$, sample gas, air: $60 \mathrm{~cm}^{3}$ $\min ^{-1}$ ) and air (balance gas, nitrogen: $40 \mathrm{~cm}^{3} \mathrm{~min}^{-1}$, sample gas, air: $60 \mathrm{~cm}^{3} \mathrm{~min}^{-1}$ ).

Dynamic mechanical thermal analysis (DMTA) experiments were run on the TA Instruments Q800 DMA. Samples $(35 \times 12 \times$ $3 \mathrm{~mm}^{3}$, cross sectional area $36 \mathrm{~mm}^{2}$ ) were oscillated in a dual cantilever clamp in a three-point flexural mode at a fixed frequency, of $1 \mathrm{~Hz}(0.1 \%$ strain $)$ while being scanned over a temperature range of -30 to $200{ }^{\circ} \mathrm{C}$. The heating rate for DMTA experiments was $4 \mathrm{~K}$ per minute and the samples were heated under a nitrogen atmosphere $\left(40 \mathrm{~cm}^{3} \mathrm{~min}^{-1}\right)$.

Mechanical measurements were made using an Instron 6600 tensile tester accompanied by a $25 \mathrm{~mm}$ (occasionally $50 \mathrm{~mm}$ ) extensometer on injection moulded 'dogbone' samples $(3 \mathrm{~mm} \times 12 \mathrm{~mm} \times 25 \mathrm{~mm})$. The crosshead speed was $1 \mathrm{~mm}$ per minute and the maximum load was recorded against time as well as strain at $1 \%$. The experiment was concluded when the material began to undergo 'necking' and the peak at maximum load began to fall. At least 10 replicate samples were analysed in each case.

Surface wetting was measured on excess material removed from the injection moulded samples used for tensile measurements by determining contact angles using a Kruss 'EasyDrop' goniometer using specialist software. All samples were cleaned with isopropyl alcohol prior to analysis and placed on the moveable stage in the goniometer. A drop of water (deposited by syringe) was placed on the polymer surface and the readings were taken after a period of stabilisation (15 seconds) and for a further 15 seconds. The contact angle was recorded at the point at which the graph begins to deviate; 10 replicate analyses were performed in each case (at a variety of points on the sample's surface) and the average number recorded.

Scanning electron microscopy (SEM) was performed using a Hitachi TM3000, coupled with a Bruker Quantax 70 for the collection of energy dispersive X-ray (EDX) spectroscopic data. Sample sections for the EDX analysis were taken at $15 \mathrm{keV}$ and the acquisition period was 120 seconds.

For the analysis of the fracture surfaces using scanning electron microscopy (SEM), the sample was nicked, before being submerged in liquid $\mathrm{N}_{2}$ for approximately 25 minutes. It was removed and fractured as quickly as possible, before being fixed to an SEM stub and coated with chromium (Emitech k575x Peltier Cooled). Three coatings of $1.5 \mathrm{~nm}$ were used, with a sputter time of 40 seconds and a $150 \mathrm{~mA}$ clean current. The sample was then warmed to room temperature and taken to the SEM (JSM-7100F), before being mounted and inserted into the specimen chamber. The fracture surface was imaged using the prescribed setting for 'coated plastic sample', with an accelerating voltage of either $1 \mathrm{kV}$ or $5 \mathrm{kV}$, at magnification of $10000 \times$.

\section{Molecular modelling}

Modelling was performed using Materials Studio v.6.0.0 (Accelrys, 2012) on a server (CHEM-4, an 8 core Xenon, $2.33 \mathrm{GHz}$ with 8 GB RAM running Windows server 2008, 64 bit). Potential energies were calculated using the COMPASS Force Field. ${ }^{8}$ Polypropylene chains were built up using the polymer builder using isotactic sequences of monomers. 10 repeat units were joined to form chains. POSS reagents were built manually and minimised before use. The required amount of POSS (5 wt\%) was added to the PP chains and the latter were packed into cells using the amorphous cell module to reproduce the literature values for the density of PP $\left(0.900 \mathrm{~g} \mathrm{~cm}^{-3}\right)$. The resulting amorphous cells comprised between 8178 and 11284 atoms. A minimization of 500 iterations was carried out to relieve the strain using the Discover minimization module to a convergence of $1000 \mathrm{kcal} \mathrm{mol}^{-1} \AA^{-1}$. A conjugate gradients algorithm was carried out to a convergence of $100 \mathrm{kcal} \mathrm{mol}^{-1} \AA^{-1}$. The legacy temperature cycle in the amorphous cell module was used for the molecular dynamics simulation. The NPT ensemble with a time step of $1 \mathrm{fs}$ was utilized with the Anderson thermostat in combination with the Berendsen barostat. ${ }^{9}$ PCFF was used with the atomic van der Waals summation, a cut-off of $10.00 \AA$, a spline width of $3.00 \AA$ and a buffer width of $1.00 \AA .51$ MD simulations were run between $77^{\circ} \mathrm{C}$ and $-23^{\circ} \mathrm{C}$ in decrements of $10 \mathrm{~K}$; at each temperature stage a 50 ps MD simulation was carried out. The first 25 ps of each simulation were used to equilibrate the system and the subsequent 50 ps simulation was used to record the results. A plot of calculated cell density was plotted against simulation temperature to determine both the $T_{\mathrm{g}}$ and the degradation onset temperature.

\section{Results and discussion}

\section{Selection and incorporation of the POSS reagents into polypropylene}

The selection of the POSS reagents was made in order to ensure the greatest degree of compatibility between matrix and additive, while reducing the hydrophobicity of the blend. Unsurprisingly, literature studies ${ }^{\mathbf{1 0}}$ have shown that the dispersion of POSS reagents into the PP matrix and the resulting morphology is often influenced by PP chain length and the structural similarity between the polymer and the POSS reagent. From an extensive catalogue of POSS reagents, those containing isobutyl and isooctyl residues bonded to the silicon atoms at the apices. At the same time, the selection of the functional groups was made in order that the ability to introduce an alcohol (AL0130) directly into the PP could be compared with a latent alcohol (analogous to a synthon as an epoxide, EP0419, or tri-epoxide, EP0423). The reasoning being that the less polar epoxide rings could be introduced and dispersed more readily in the non polar PP, before undergoing ring opening during the molten blending process to introduce sites for hydrogen bonding. 

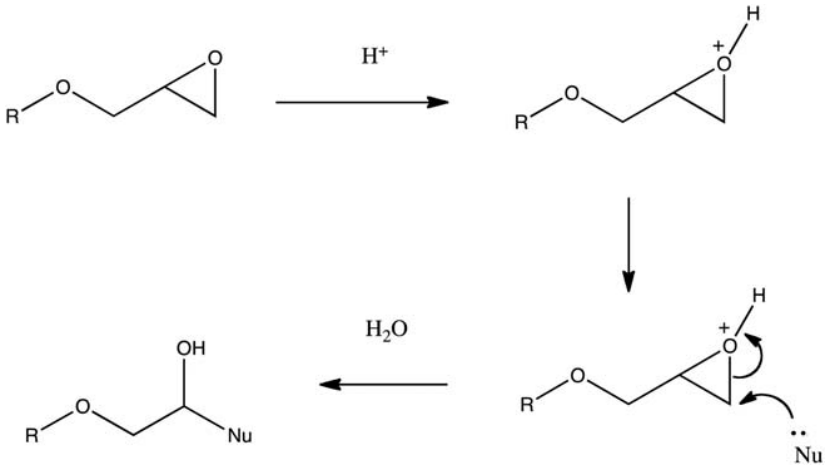

Fig. 2 General mechanism for the acid catalysed ring opening of the epoxide group.

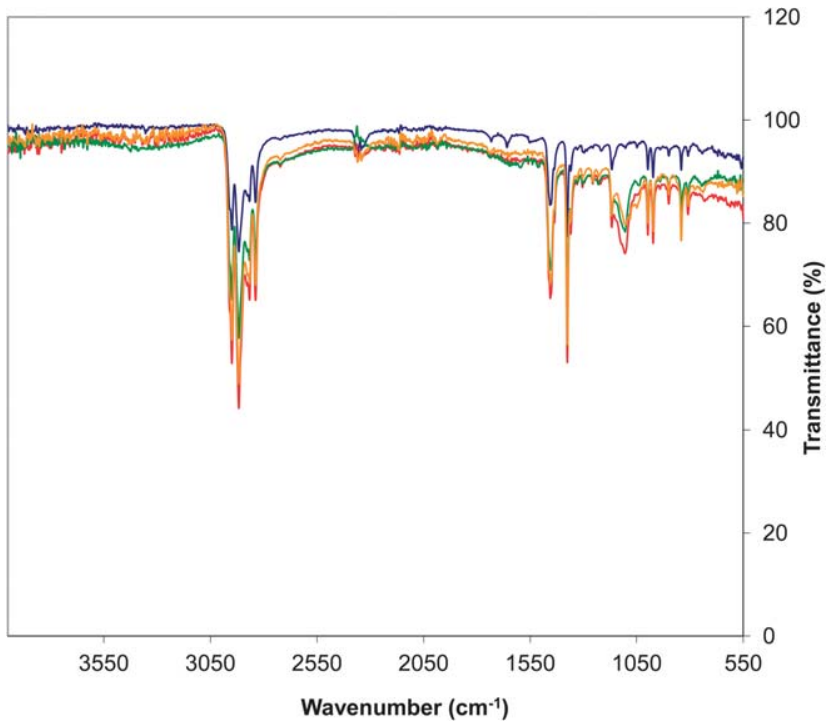

Fig. 3 Infrared spectra (recorded in transmission) of (a) PP standard (blue), (b) PP/EP0419 (red), (c) PP/AL0130 (green), and (d) PP/EP0423 (gold).

Epoxy resins, particularly in the form of glycidyl ethers, are known to be sensitive to both acid- and base-catalysed ringopening reactions with mild heating and ultraviolet radiation. ${ }^{11,12}$ Owing to the geometry of the oxirane moiety, in which the 3-membered ring is highly strained, this can be accomplished at comparatively low temperatures (i.e. during the blending step) in the presence of adventitious moisture or even with residual polymerisation catalyst in the PP (Fig. 2), where there is the possibility of nucleophilic species being present. As the compounding temperature zones during extrusion ranged from 150 to $180{ }^{\circ} \mathrm{C}$, the ring-opening reaction would be not only favoured but rapid (in a previous study ${ }^{13}$ the diglycidyl ether of bisphenol A displayed a $k_{1}$ value of $4.48 \times$ $10^{-4} \mathrm{~s}^{-1}$ at $150{ }^{\circ} \mathrm{C}$ for the oxirane ring opening reaction in the presence of 2-ethyl-4-methylimidazole).

Vibrational spectroscopy (Fig. 3) was used to confirm the incorporation of the POSS reagents prior to further thermal and mechanical analysis. According to previous studies on octamethyl-POSS, ${ }^{\mathbf{1 4 , 1 5}}$ the bands between 1000 and $1125 \mathrm{~cm}^{-1}$ are characteristic of the $\mathrm{Si}-\mathrm{O}-\mathrm{Si}$ stretches and, whilst these were absent in the PP sample (as expected), they were observed in the PP samples blended with the POSS reagents at $1103 \mathrm{~cm}^{-1}$ (AL0130), $1110 \mathrm{~cm}^{-1}$ (EP0419), and $1098 \mathrm{~cm}^{-1}$ (EP0423) respectively.

Raman spectroscopy was also used in a complementary fashion to observe $\mathrm{Si}-\mathrm{O}-\mathrm{Si}$ deformations at $453,532 \mathrm{~cm}^{-1}$ (AL0130), 451, $524 \mathrm{~cm}^{-1}$ (EP0419), and 451, $523 \mathrm{~cm}^{-1}$ (EP0423) respectively. Some indication of the presence of $\mathrm{OH}$ groups were found using infrared through weak bands (3550-3250 $\mathrm{cm}^{-1}$ ), suggesting that the even the epoxy-based POSS reagents (EP0419 and EP0423) had indeed undergone ring opening during the blending process. This is a useful attribute since the epoxides (being significantly less polar than the alcohol) should be somewhat easier to introduce into the $\mathrm{PP}$, prior to ring opening to yield hydroxylation.

The degree of dispersion of nanofillers plays an important role in influencing the properties of the resulting nanocomposites and energy dispersive X-ray spectroscopy (EDX), combined with scanning electron microscopy, was employed to analyse the elemental composition on the virgin surface and following microtoming through the bulk of the sample. With a PP matrix, comprising carbon and hydrogen, the presence of oxygen and silicon (Fig. 4) offers a good measure of the presence

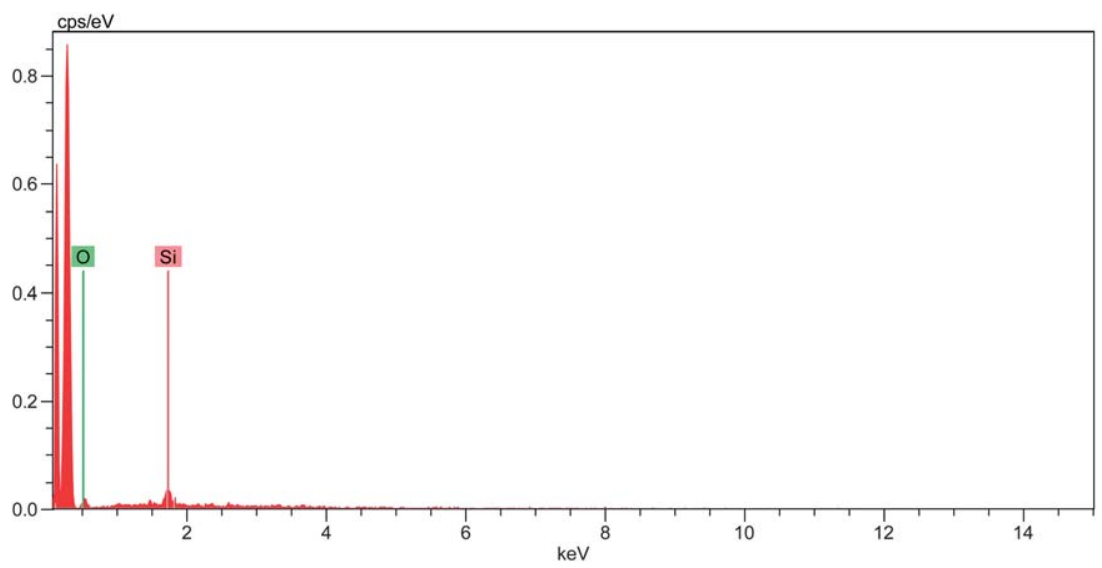

Fig. 4 EDX spectrum of the virgin surface of the PP nanocomposite containing 5 wt\% AL0130. 
of the POSS reagents (not only in the cage, but also the glycidyl or hydroxylated functional groups).

Further analysis of the bulk surface involved the progressive removal of around 300 microns of the matrix to produce a crude depth profile (Fig. $5 \mathrm{a}-\mathrm{c}$ ). The evidence of the elemental analysis

(a)

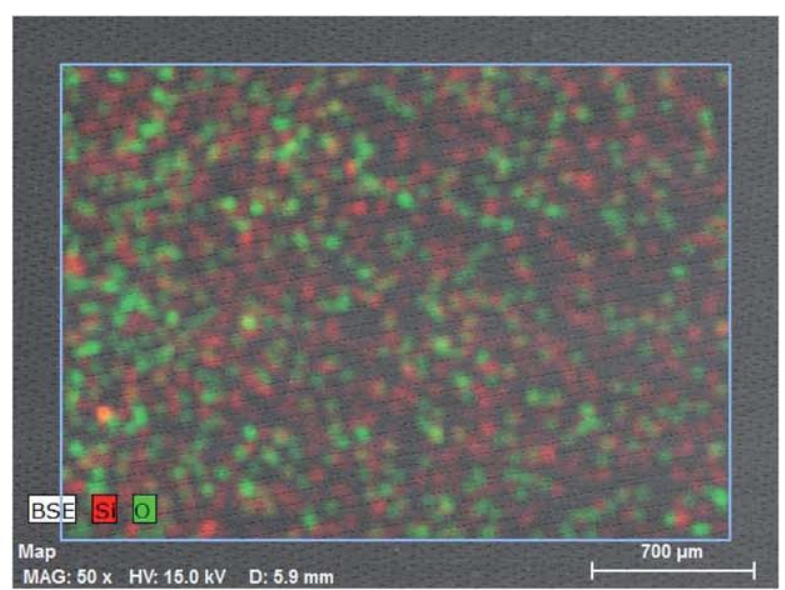

(b)

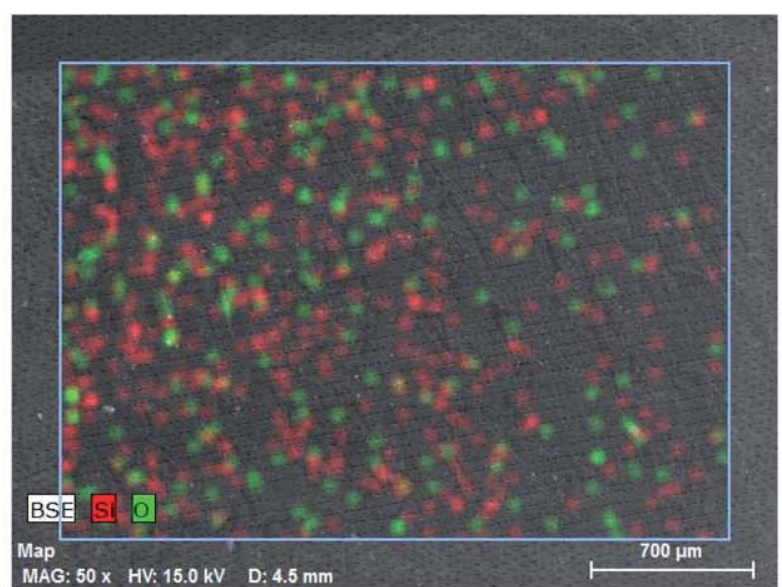

(c)

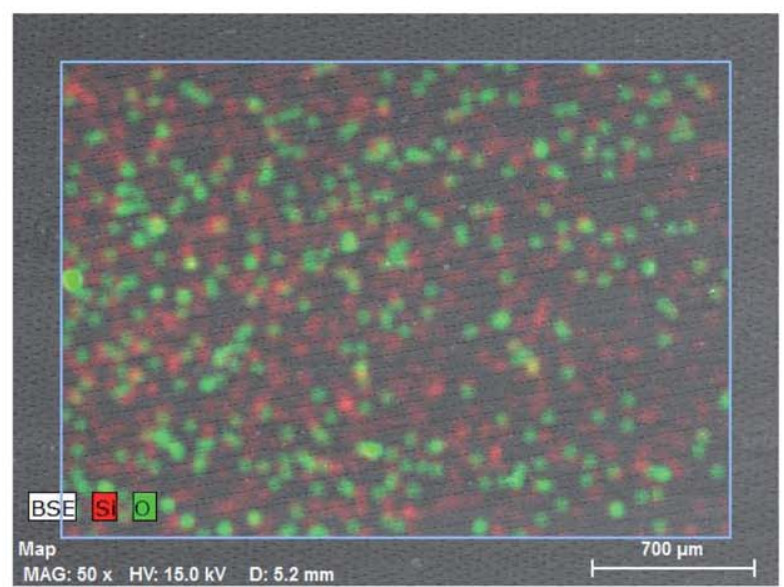

Fig. 5 (a) Elemental maps, magnification $50 \times$ from SEM-EDX of (a) the virgin surface of the PP/AL0130 nanocomposite showing the distribution of silicon (red) and oxygen (green) atoms (b) following removal of $10 \%$ of the surface depth, (c) following removal of $50 \%$ of the surface depth of the bulk polymer. suggests that the nanofiller is well dispersed within and throughout the bulk PP matrix, supporting the observation that the blending process was thorough and effective.

At the same time the samples were submerged in liquid $\mathrm{N}_{2}$ before being fractured to expose fresh surfaces. The latter were coated using chromium prior to analysis using SEM and representative images (magnification $10000 \times$ ) are shown for two different nanocomposites when subjected to accelerating voltages of $1 \mathrm{kV}$ (Fig. 6a) and $5 \mathrm{kV}$ (Fig. 6b); the analysis of the other samples resulted in similar images. The sample bar is $1 \mu \mathrm{m}$ indicating that any agglomerates present are sub micron in size.

Although the dispersion of the POSS reagents appears to be relatively uniform, differential scanning calorimetry (DSC) was also employed to demonstrate the homogeneity of the nanocomposites, specifically through examination of the melting behaviour. The DSC thermograms (Fig. 7) for the rescan experiments for each of the samples under examination show the effect of introducing the POSS reagents. The melting endotherm observed for PP commences at a relatively low temperature $\left(100{ }^{\circ} \mathrm{C}\right)$, but results in a comparatively sharp melting temperature (peak minimum $151{ }^{\circ} \mathrm{C}$ at $10 \mathrm{~K} \mathrm{~min}^{-1}$ ) accounting for $\Delta H_{\mathrm{m}}=71 \mathrm{~J} \mathrm{~g}^{-1}$ indicating the presence of both crystalline and amorphous domains in the PP matrix. The enthalpy of melting compares favourably with other published values for isotactic PP. ${ }^{16}$ The presence of POSS reagents serves to
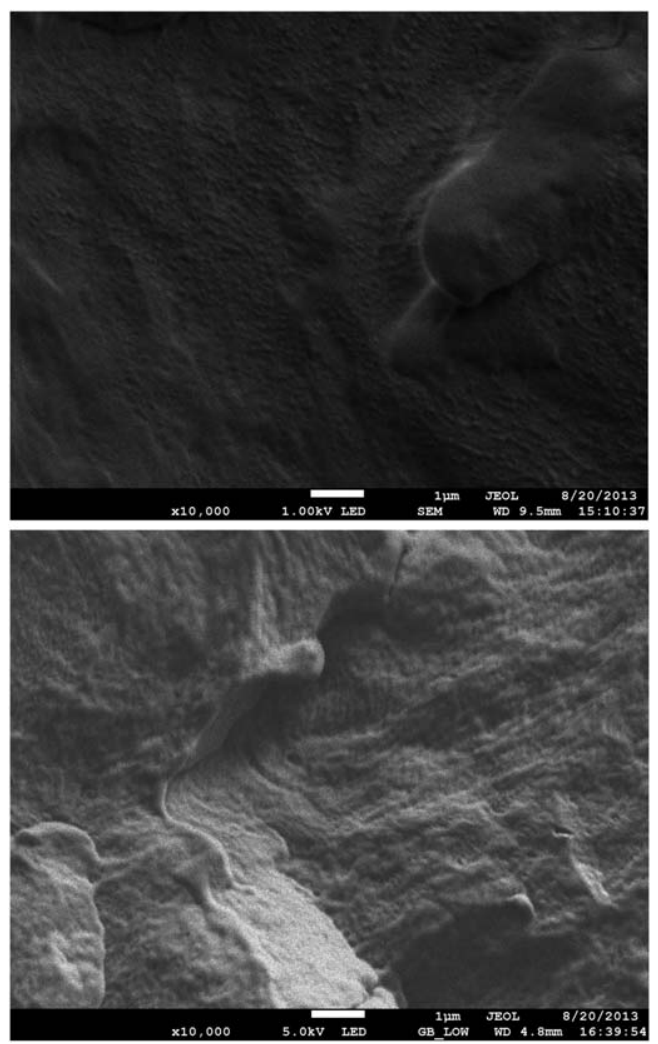

Fig. 6 SEM micrographs (magnification $10000 \times$ ) of fracture surfaces of (a) PP/ EP0423 using a beam voltage of $1 \mathrm{kV}$ and (b) PP/AL0130 using a beam voltage of $5 \mathrm{kV}$. 


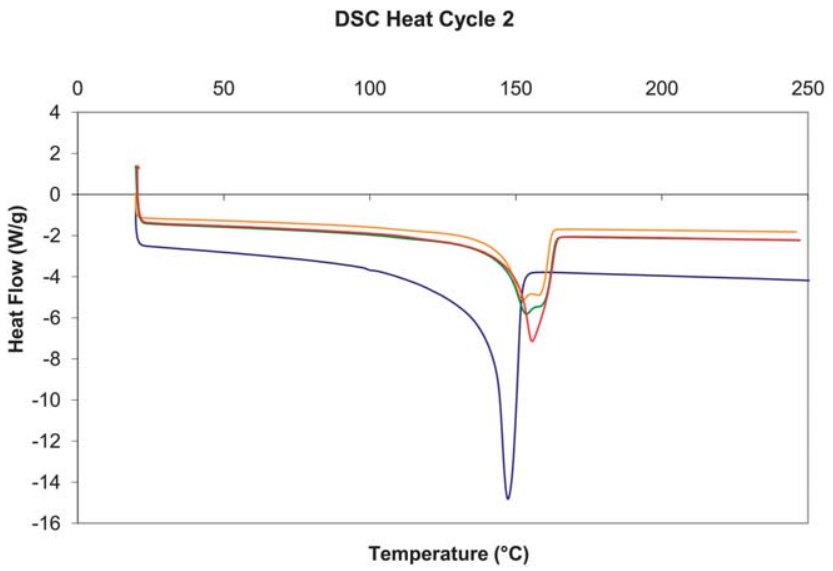

Fig. 7 Differential scanning data ( $\mathrm{W} \mathrm{g}^{-1}$ versus temperature, Exo upwards) for the second heating experiment (heat/cool/heat from $20{ }^{\circ} \mathrm{C}$ to $250{ }^{\circ} \mathrm{C}$ at $10 \mathrm{~K}$ $\mathrm{min}^{-1}$, under nitrogen) for (a) PP standard (blue), (b) PP/EP0419 (red), (c) PP/ AL0130 (green), and (d) PP/EP0423 (gold).

increase the melting temperature very slightly and yield two overlapping endothermic peaks, although the $\Delta H_{\mathrm{m}}$ appears to undergo a slight reduction $\left(64-65 \mathrm{~J} \mathrm{~g}^{-1}\right.$, peak minimum $158^{\circ} \mathrm{C}$ ) meaning that processing appears little affected by the addition of the additive at this level. The thermogram shown in Fig. 7b (for PP/EP0419) appears to represent the most homogeneous blend with the simplest endotherm (with less bimodal character).

In this preliminary study, the nanofillers were simply blended with the commercial polyolefin, without concentrating on employing reactive compatibilisation. Other published studies ${ }^{17}$ have demonstrated that the use of PP-methyl POSS as a compatibiliser within a PP matrix can yield enhancements in mechanical performance and Fina et al. ${ }^{\mathbf{1 8}}$ have comprehensively reviewed the area of POSS-based hybrids produced through melt/reactive blending in great depth. Indeed, we have employed this approach in a similar fashion to produce linkedinterpenetrating networks in thermoset matrices with enhanced physical properties and improved hydrolytic stability. ${ }^{\mathbf{1 9 2 0}}$ Nevertheless, the nanofillers appear to be well dispersed within the thermoplastic matrix and attention turned to the properties of the nanocomposites.

\section{Examination of the influence of nanofillers on the thermal and mechanical behaviour of the PP/POSS nanocomposites}

Although the main thrust of the work was to modify the hydrophobicity of the resin matrices, it was key that the existing physical and mechanical properties of the isotactic PP should not be degraded in the process. Having examined the homogeneity of the nanocomposites using DSC, the thermomechanical behaviour of the materials was examined using DMTA. This was intended as a preliminary study to examine the $T_{\mathrm{g}}$ behaviour since the effect of the POSS on tensile modulus would be determined with greater rigour using 'dogbone' samples. The data for the storage modulus for both PP and the nanocomposites are shown in Fig. 8.

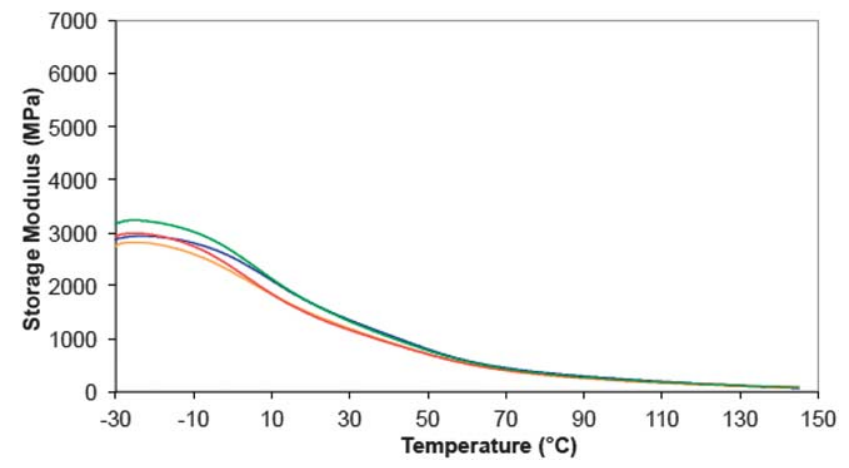

Fig. 8 DMTA data shown as storage modulus ( $E^{\prime}, \mathrm{MPa}$ ) (dual cantilever, $1 \mathrm{~Hz}$, $0.1 \%$ strain) for (a) PP standard (blue), (b) PP/EP0419 (red), (c) PP/AL0130 (green), and (d) PP/EP0423 (gold). All experiments were performed from $-30^{\circ} \mathrm{C}$ to $150^{\circ} \mathrm{C}$ at $10 \mathrm{~K}$ per minute, in nitrogen.

All of the samples display similar features: the PP displays a drop in the storage modulus at $c a .5-9{ }^{\circ} \mathrm{C}$, consistent with the $T_{\mathrm{g}}$ and the area under the thermogram (loss modulus, not shown) is $c a$. $247 \mathrm{MPa} \min ^{-1}$; a second reduction in the loss modus occurs at around $30-70{ }^{\circ} \mathrm{C}$. The introduction of AL0130 reduces the peak in the loss modulus (the $T_{\mathrm{g}}$ ) and the area under the thermogram rises to $c a$. $302 \mathrm{MPa} \mathrm{min}^{-1}$; the second reduction in the loss modus appears unchanged. This is seen in the context of all of the nanocomposites in Fig. 8, from which it can be seen that $T_{\mathrm{g}}$ is most affected by the introduction of EP4019 and EP4023, although it is not clear from this study whether this is due to a change in morphology. Molecular simulations were performed over a wider range of temperature than is our normal habit in order to capture not only low temperature mechanical motions (as evidenced by the DMTA measurements), but also processes that might herald the onset of thermal degradation. Fig. 9 shows the results of the temperature ramped molecular dynamics simulation for PP/EP0423. The plot clearly shows a change in the density of the model between 0 and $50{ }^{\circ} \mathrm{C}$, which corresponds closely with the experimental value determined using DMTA; the results of the other simulations all yielded $T_{\mathrm{g}}$

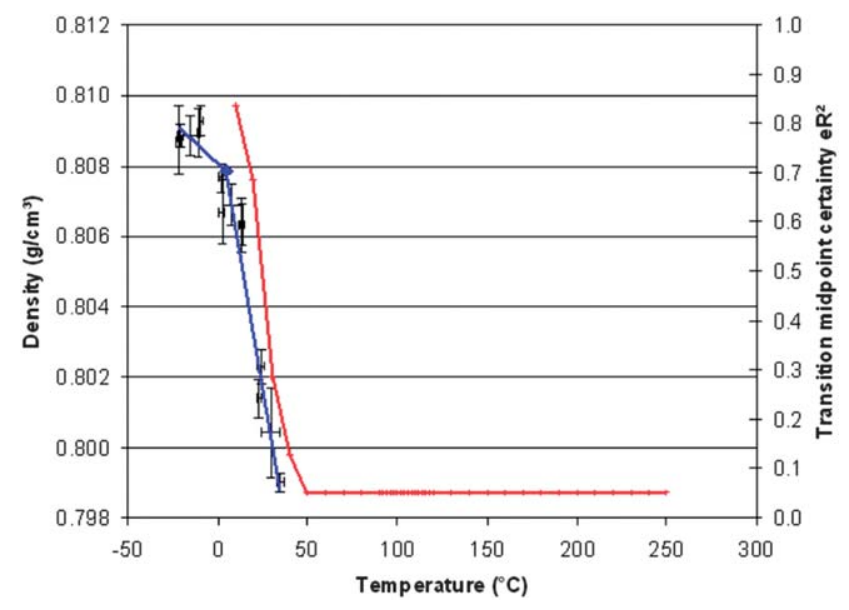

Fig. 9 Plot of simulated density versus temperature for PP/EP0423. 
values of $10^{\circ} \mathrm{C}$ (Table 1$)$. The experimentally measured values of $T_{\mathrm{g}}$ all fall between 0 and $10{ }^{\circ} \mathrm{C}$, i.e. for PP $9{ }^{\circ} \mathrm{C}, \mathrm{PP} / \mathrm{AL} 01305^{\circ} \mathrm{C}$, $\mathrm{PP} / \mathrm{EP} 04192{ }^{\circ} \mathrm{C}, \mathrm{PP} / \mathrm{EP} 04231{ }^{\circ} \mathrm{C}$, indicating that the incorporation of these POSS reagents selected in this study has little effect on the observed $T_{\mathrm{g}}$ values (at a level of $5 \mathrm{wt} \%$ ) and the simulations also show this insensitivity to change in $T_{\mathrm{g}}$.

Preliminary measurements of the thermal and thermooxidative stability of the PP were made using TGA as a measure of internal consistency and the thermograms are shown in Fig. 10.

The data presented for the unmodified PP are in agreement with published literature: the onset of degradation in air occurs at $c a .240{ }^{\circ} \mathrm{C}$ and is almost complete by $340{ }^{\circ} \mathrm{C}$; onset commences at $450{ }^{\circ} \mathrm{C}$ in nitrogen and is virtually complete by $475^{\circ} \mathrm{C}$ at this heating rate (lit. $475^{\circ} \mathrm{C}$ (ref. 21)). The introduction of the POSS reagents to form the nanocomposites gave rise to a slight reduction (and more gradual mass loss) in the initial onset of degradation. However, the most significant enhancement occurs at temperatures above $341{ }^{\circ} \mathrm{C}$, where the completion of degradation is delayed, although at these relatively low concentrations this is not particularly marked. This finding is in line with other reports in which the introduction of phenylsubstituted POSS, delayed the completion of degradation from $341{ }^{\circ} \mathrm{C}$ to between 350 and $376{ }^{\circ} \mathrm{C}$, depending on the concentration employed. ${ }^{22}$ Furthermore, the profile the thermal degradation serves to confirm that the apparent mechanism

Table 1 Simulated thermal properties of the PP/POSS nanocomposites

\begin{tabular}{lll}
\hline POSS reagent & $T_{\mathrm{g}}\left({ }^{\circ} \mathrm{C}\right)$ & $T_{\mathrm{d}}\left({ }^{\circ} \mathrm{C}\right)$ \\
\hline AL0130 & 10 & 200 \\
EP0419 & 10 & 230 \\
EP0423 & 10 & 200
\end{tabular}

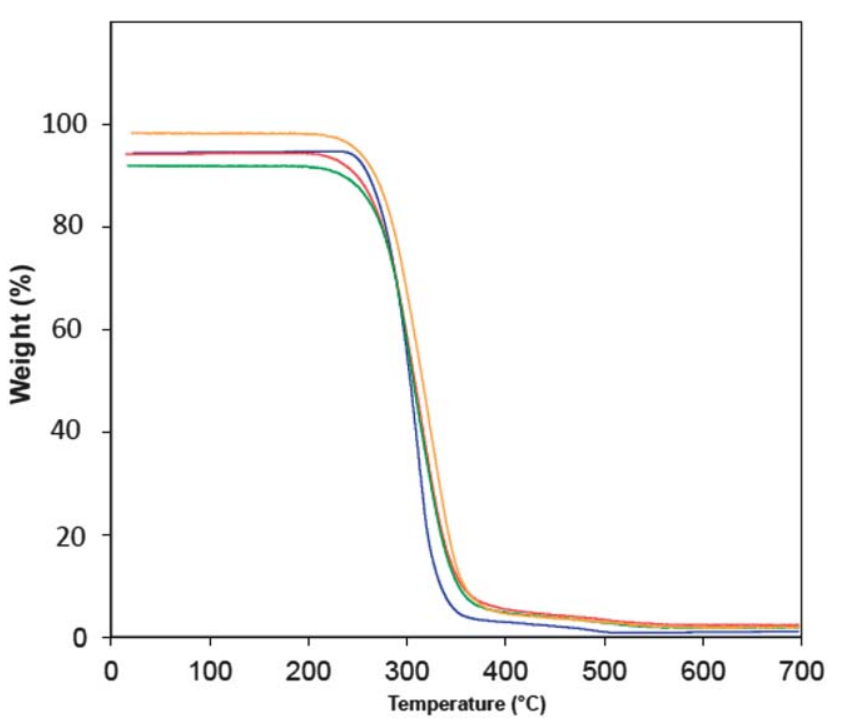

Fig. 10 TGA data for (a) PP standard (blue), (b) PP/EP0419 (red), (c) PP/AL0130 (green), and (d) PP/EP0423 (gold). All experiments were performed in air from room temperature to $700{ }^{\circ} \mathrm{C}$ at $10 \mathrm{~K}$ per minute. does not appear to vary significantly in the early stages of decomposition. Temperature ramped molecular dynamics simulation was also employed to probe the onset of thermal degradation $\left(T_{\mathrm{d}}\right)$ - the latter is a comparatively recent. ${ }^{23}$ addition to our panoply of simulation techniques and has been employed with some success to examine the degradation behaviour of thermosetting polymers such as cyanate esters, ${ }^{24}$ polybenzoxazines, ${ }^{25}$ and epoxy resins. ${ }^{26}$ TGA revealed that $T_{\mathrm{d}}$ was found empirically to be relatively insensitive to incorporation of POSS at this level and this again borne out by the simulations, which indicates that there are no interactions taking place in the nanocomposites that are not accounted for in the model.

During the production of the dogbone samples for the tensile measurements, initial visual observations suggested that the incorporation of POSS reagents into the PP led to a reduction in the thermo-oxidative stability. The translucent white pellets became slightly more brown in colour during the injection moulding process and increased the degree of shrinkage, which is in keeping with the TGA data for the onset of degradation. At least 7 samples of each material were tested in this fashion and the data are presented in Table 2.

In addition to the data presented, the PP base polymer took ca. 10 minutes to fail, compared with 15-20 minutes for the PP/ POSS nanocomposites; the maximum load was also reduced significantly on the addition of the POSS reagents, with the greatest reduction observed for the triglycidyl ether. The UTS data for the base polymer show good agreement with other studies (e.g. 24.8 MPa (ref. 27) and 22.2 MPa (ref. 28)) validating the comparison study. In this work the introduction led to a small reduction in ultimate tensile strength, while tensile modulus appeared little changed. In other studies Young's modulus has been found to be influenced by the nature of the POSS reagent being incorporated e.g., the introduction of octamethyl POSS led to an increase in this property in PP, whereas octa-isobutyl or octa-isoctyl POSS had the opposite effect. ${ }^{29}$ This was postulated to be due to the lengthy alkyl chains enveloping the hard, silicon cage and constituting a softer outer shell, which might limit the stress transfer from the polymer matrix to the hard core. This is less marked in our own work, but appears to hold for the maximum load in which the POSS reagents containing isobutyl groups (AL0130 and EP0419) have similar values, but are markedly greater than the isooctyl residue (EP0423).

\section{Examination of the wetting behaviour and hydrophobicity of the PP/POSS nanocomposites}

In summary, the incorporation of the POSS reagents had demonstrably yielded PP nanocomposites with similar thermal and mechanical properties to the unmodified isotactic PP homopolymer. On this basis, the effect of the addition of POSS reagents on the wetting behaviour of the PP through contact angle measurements was examined. The surface free energy is effectively a measure of the hydrophobicity of a material, and is calculated by using an obtained value of the contact angle. The lower the contact angle, the higher the surface free energy and 
Table 2 Tensile properties of the PP and PP/POSS nanocomposites ${ }^{a}$

\begin{tabular}{|c|c|c|c|c|}
\hline Sample & UTS (MPa) & $\mathrm{TM}(\mathrm{MPa})$ & Maximum load (N) & Strain at $1 \%(\mathrm{GPa})$ \\
\hline $\mathrm{PP}$ & $24.73 \pm 0.10$ & $13.51 \pm 1.55$ & $890.12 \pm 3.76$ & $1.24 \pm 0.06(n=9)$ \\
\hline PP/AL0130 & $21.95 \pm 0.10$ & $10.23 \pm 0.29$ & $790.04 \pm 13.38$ & $1.23 \pm 0.03(n=8)$ \\
\hline PP/EP0419 & $22.52 \pm 0.44$ & $11.03 \pm 0.72$ & $810.76 \pm 15.67$ & $1.03 \pm 0.03(n=7)$ \\
\hline PP/EP0423 & $21.06 \pm 0.28$ & $10.23 \pm 1.13$ & $758.15 \pm 10.03$ & $1.02 \pm 0.13(n=10)$ \\
\hline
\end{tabular}

${ }^{a}$ Key: UTS $=$ ultimate tensile strength, TM $=$ tensile modulus.

the less hydrophobic a material. This relationship was first established by Young in 1805 and the resulting equation (eqn (1)), or a derivation of it known as "Young's Equation" (eqn (2) $),{ }^{30}$ is employed in many commercial instruments that quantify surface free energy.

$$
\begin{aligned}
& W_{\mathrm{a}}=\gamma_{\mathrm{S}}+\gamma_{\mathrm{L}}-\gamma_{\mathrm{SL}} \\
& \gamma_{\mathrm{L}} \cos \theta=\gamma_{\mathrm{S}}-\gamma_{\mathrm{SL}}
\end{aligned}
$$

Young's equation considers a water droplet on a solid surface and interfacial tensions at three locations: $\gamma_{\mathrm{L}}$ (the liquid-vapour interface), $\gamma_{\mathrm{S}}$ (the solid-vapour interface) and $\gamma_{\mathrm{SL}}$ (the solid-liquid interface). It is derived by taking into account all forces acting along the line of contact, which at equilibrium must cancel each other out and result in a net figure of zero. Practically, the determination of contact angles with precision is known to present challenges (only $\gamma_{\mathrm{L}}$ can be measured as the surface tension of the solid can be determined experimentally only against a series of known liquids ${ }^{31}$ ) and the data reported are often hotly disputed. From the studies that have been conducted, it is clear that in order to collect data most reliably it is key that the surface must not be too rough, as a superhydrophobic environment may be formed (and $\gamma_{\mathrm{SL}}$ will be heavily dependent on the contact angle formed). Unfortunately, during this work it was not possible to gain access to dynamic measurements, although the authors accept that there would be merit in evaluating the advancing and receding contact angles, from which the extent of any hysteresis might be determined. The collected data for the standard PP samples and the nanocomposites are shown in Table 3.

The relatively high contact angle of the PP standard results from its lack of hydrogen bonding sites and great number of van der Waals interactions. For instance, a strongly hydrophobic surface will display a contact angle in excess of $90^{\circ}$; a strongly hydrophilic surface $\ll 90^{\circ}$ and more commonly moderately polar surfaces fall somewhere in between. Thus, in this study the strongly hydrophobic PP standard was rendered slightly less hydrophobic with the incorporation of either triglycidylisobutyl

Table 3 Comparison of contact angles $\left({ }^{\circ}\right)$ of PP and PP/POSS nanocomposites

\begin{tabular}{lrlll}
\hline Sample & PP & PP/AL0130 & PP/EP0419 & PP/EP4023 \\
\hline 1 & 100.54 & 86.47 & 94.56 & 86.86 \\
2 & 99.49 & 88.75 & 94.27 & 84.22 \\
3 & 99.13 & 88.99 & 97.11 & 86.23 \\
Average & 99.72 & 85.77 & 95.32 & 88.07
\end{tabular}

POSS (EP4023), which would be present as a triol following extrusion, or 1,2-propanediolisobutyl POSS (AL0130).

An approach involving quantitative structure property relationships (QSPR) was investigated to probe the hydrophobicity of the surface and relate this to the nature of the POSS reagents that had been selected. The ultimate aim of this aspect of this preliminary work would be to yield a model capable to predicting the most suitable POSS reagent to yield favourable wetting properties whilst maintaining desirable physical and mechanical performance. The QSPR method was initially pioneered by Van Krevelen culminating in a book published $^{32}$ in 2009 and in previous publications we have reported the use of QSPR to predict variously the $T_{\mathrm{g}}$ values of poly(arylene ether sulphone)s, ${ }^{33,34}$ char yield ${ }^{35}$ and thermal properties $^{36}$ of polybenzoxazines. QSPR works well when one has access to a large dataset of compounds, but in this case we have effectively four experimental points, the unmodified polymer and the three POSS additives. When there are only three experimental points then a linear fit is always possible, so we are just barely in the regime where partial least squares (PLS) is applicable. With a scarcity of data many possible equations are potential solutions, so we have taken the approach of fitting to the minimum number of parameters and chosen the parameters that we want to fit to carefully so that they will provide information on the structural properties of the POSS materials. It is known that the inherent hydrophilicity in polymer structures results from a combination of several parameters, notably hydrogen bonding opportunities per unit length and the number of van der Waals interactions. Thus, the method was to perform multiple linear regression on all the data available to include the accessible surface area, the hydrogen bond acceptor count, and the van der Waals (non bonded energy); the parameters were calculated (Table 4) with the Moe 2011.10 program. ${ }^{37}$

The parameters were chosen carefully on the basis of knowledge of the factors that generally affect the chosen physical properties of the polymers (i.e. tensile modulus, $T_{\mathrm{g}}$, and the

\begin{tabular}{|c|c|c|c|}
\hline POSS reagent & $\begin{array}{l}\text { Accessible } \\
\text { SA }\left(\mathrm{m}^{2}\right)\end{array}$ & HB acceptor count & $\begin{array}{l}\text { vdW energy (kcal } \\
\mathrm{mol}^{-1} \text { ) }\end{array}$ \\
\hline AL0130 & 1136.3 & 3 & 28.2 \\
\hline EP0419 & 1727.5 & 2 & 90.2 \\
\hline EP0423 & 1538.5 & 6 & 25.4 \\
\hline
\end{tabular}

Table 4 Selected properties of the POSS reagents ${ }^{a}$

${ }^{a}$ Key: accessible $\mathrm{SA}=$ accessible surface area, $\mathrm{HB}$ acceptor count $=$ hydrogen bond acceptor count, vdW energy = van der Waals energy. 
maximum load to break). The hydrogen bond acceptor count represents the ability of the POSS reagent to form hydrogen bonds. The tensile modulus scales with the accessible surface area of the POSS reagents: a more open structure giving the highest value of the tensile modulus. The load to break depends upon the non-bonded energy, with the largest values seen for the POSS reagents having the greatest portion of their structures contributing to van der Waals interactions. Likewise the UTS depends strongly on the van der Waals interaction energy. As this arises because of dispersion forces and the formation of induced dipoles, having a strongly polar moiety in the structure serves to enhance this interaction and conversely reduces the UTS slightly from that of PP. The regression equations were performed by partial least squares in Moe selecting the one parameter that gave the largest correlation coefficient. This result suggests that the selection of a particular POSS reagent to optimise a given parameter might be routinely possible from calculation of just three properties from molecular modelling. Work is currently underway to extend this study further and select more suitable POSS reagents based on the QSPR approach explored herein and will be reported in a future publication.

\section{Conclusions}

The addition of comparatively small quantities (5 wt\%) of a variety of POSS reagents to isotactic PP is possible without degrading the existing properties of the polymer too greatly (there is a small impact on $T_{\mathrm{g}}$ and the maximum load recorded). Given the polarity of the additive, there is a practical limit to the amount of POSS that can be introduced to a polyolefin. However, the reduction in hydrophobicity possible is significant (a reduction in contact angle of some $14^{\circ}$ is observed) with the use of a triglycidylisobutyl-POSS. This reagent offers the benefit of being introduced and dispersed in its glycidyl ether form, but undergoes ring opening (the oxirane rings are extremely strained) to yield three hydroxyl groups per POSS molecule. The QSPR modelling shows that the tensile properties of the PP/ POSS nanocomposites depend strongly on the accessible surface area of the POSS moieties and their electronic interactions. The Molecular simulation shows that it is possible to model these composites and produce simulated thermal properties that are congruent with literature values. The success of the simulation approaches confirms the power of the approach and consequently these methods will be used to predict the properties of $\mathrm{PP} / \mathrm{POSS}$ nanocomposites using other POSS reagents prior to procurement and blending/analysis.

\section{Acknowledgements}

We thank Dr Joe Lichtenhahn (Hybrid Plastics, Hattiesburg, USA), for advice given during the procurement of the POSS reagents, Mr Toby Kemp (Instron) for assistance with the evaluation of the mechanical data, and at the University of Surrey: $\mathrm{Mr}$ Peter Haynes and Dr David Jesson (Faculty of Engineering and Physical Sciences) for facilitating access to analytical equipment, Mr Chris Burt for assistance with microscopy, and Ms. Violeta
Doukova (Surrey Materials Institute). We are particularly grateful to Professor John Watts (Faculty of Engineering and Physical Sciences) for constructive discussions about surface analysis during the preparation of the manuscript.

\section{References}

1 http:/www.ceresana.com/en/studies/market-studies/plastics/ polypropylene, accessed 23 April 2011.

2 http://www.accudynetest.com/polymer_surface_data/poly propylene.pdf, accessed 30 May, 2012.

3 O.-J. Kwon, S. Tang, S.-W. Myung, N. Lu and H.-S. Choi, Surf. Coat. Technol., 2005, 192, 1-10.

4 C. Wanke, J. Feijó, L. Barbosa, L. Campo, R. Oliveira and F. Horowitz, Polymer, 2011, 52, 1797-1802.

5 S. Kuo and F. Chang, Prog. Polym. Sci., 2011, 36, 1649-1696. 6 R. Mistra, A. Alidedeoglu, W. Jarrett and S. Morgan, Polymer, 2009, 50, 2906-2918.

7 Q. Zhou, K. P. Pramoda, J.-M. Lee, K. Wang and L. S. Loo, J. Colloid Interface Sci., 2011, 355, 222-230.

8 H. Sun, J. Phys. Chem. B, 1998, 102, 7338-7364.

9 H. J. C. Berendsen, J. P. M. Postma, W. F. van Gunsteren, A. DiNola and J. R. J. Haak, Chem. Phys., 1984, 81, 3684-3690.

10 S. Li, G. P. Simon and J. G. Matisons, Polym. Eng. Sci., 2009, 50, 991-999.

11 N. Egger, K. Schmidt-Rohr, B. Blümich, W.-D. Domke and B. Stapp, J. Appl. Polym. Sci., 2003, 44, 289-295.

12 J. V. Crivello and J. H. W. Lam, The photoinitiated cationic polymerisation of epoxy resins, in Epoxy Resin Chemistry, ACS, Washington DC, 1979, ch. 1, pp. 1-16.

13 G. J. Buist, I. Hamerton, B. J. Howlin, J. R. Jones, S. Liu and J. M. Barton, J. Mater. Chem., 1994, 4, 1793-1797.

14 A. Fina, D. Tabunai, F. Carniato, A. Franche, E. Boccaleri and G. Camino, Thermochim. Acta, 2006, 440, 36-42.

15 Y. Liu, Y. Huang and L. Liu, Compos. Sci. Technol., 2007, 67, 2864-2870.

16 F. Zhang, Y. Gong and T. He, Eur. Polym. J., 2003, 39, 23152322.

17 L. O. Hui, Z. A. Mohd Ishak and H. M. Akil, Mater. Des., 2009, 30, 748-751.

18 A. Fina, O. Monticelli and G. Camino, J. Mater. Chem., 2010, 20, 9297-9305.

19 A. Chaplin, I. Hamerton, H. Herman, A. K. Mudhar and S. J. Shaw, Polymer, 2000, 41, 3945-3956.

20 I. Hamerton, H. Herman, K. T. Rees, A. Chaplin and S. J. Shaw, Polym. Int., 2001, 50, 475-483.

21 I. Krupa and A. S. Luyt, Polym. Degrad. Stab., 2001, 71, 505-508. 22 A. Fina, D. Tabunai and G. Camino, Eur. Polym. J., 2010, 46, 14-23.

23 A. Baggott, J. R. Bass, I. Hamerton, B. J. Howlin, L. Mooring and D. Sparks, A novel method to predict thermal degradation behaviour in cyanate esters and nanocomposites using molecular dynamics simulation, 2012, submitted.

24 A. O. Crawford, B. J. Howlin, G. Cavalli and I. Hamerton, React. Funct. Polym., 2012, 72, 596-605.

25 I. Hamerton, B. J. Howlin, A. L. Mitchell, S. A. Hall and L. T. McNamara, Using molecular simulation to predict 
the physical and mechanical properties of polybenzoxazines, in Handbook of Benzoxazine Resins, ed. H Ishida and T Agag, Elsevier, Amsterdam, 2011, ch. 5, pp. 127-142.

26 S. A. Hall, B. J. Howlin, I. Hamerton, A. Baidak, C. Billaud and S. Ward, PLoS One, 2012, 7(8), e42928.

27 R. Strapasson, S. Amico, M. Pereira and T. Sydenstricker, Polym. Test., 2005, 46, 468-473.

28 A. Suarez, F. Coutihno and T. Sydenstricker, Polym. Test., 2003, 22, 819-824.

29 F. Baldi, F. Bignotti, A. Fina, D. Tabunai and T. Ricco, J. Appl. Polym. Sci., 2007, 105, 935-943.

30 T. Tadros, Applied Surfactants: Principles and Applications, John Wiley \& Sons, Morlenbach, 2005, pp. 353-355.

31 I. Prigogine and S. A. Rice, Advances in Chemical Physics, John Wiley \& Sons, New Jersey, 2005, vol. 131, p. 75.
32 D. W. van Krevelen and K. te Nijenhuis, Properties of Polymers. Their Correlation with Chemical Structure, Their Numerical Estimation and Prediction from Additive Group Contribution, Elsevier, Amsterdam, 2009.

33 I. Hamerton, B. J. Howlin and V. Larwood, J. Mol. Graphics, 1995, 13, 14-17.

34 I. Hamerton, B. J. Howlin and G. Kamyszek, PLoS One, 7(6), e38424, DOI: 10.1371/journal.pone.0038424.

35 P. Mhlanga, W. A. Wan Hassan, I. Hamerton and B. J. Howlin, Polym. Degrad. Stab., 2013, 98, 446-452.

36 P. Mhlanga, W. A. Wan Hassan, I. Hamerton and B. J. Howlin, PLoS One, 8(1), e53367, DOI: 10.1371/ journal.pone.0053367.

37 Chemical Computing Ltd., http:/www.chemcomp.com/, accessed 25 May, 2012. 